The AstrophysiCAL JouRnAL, 432: L87-L90, 1994 September 10

(C) 1994. The American Astronomical Society. All rights reserved. Printed in U.S.A.

\title{
TWO-SIDED EJECTION IN POWERFUL RADIO SOURCES: THE COMPACT SYMMETRIC OBJECTS
}

\author{
P. N. Wilkinson and A. G. Polatidis \\ University of Manchester, Nuffield Radio Astronomy Laboratories, Jodrell Bank, Macclesfield, Cheshire SK11 9DL, UK
}

AND

A. C. S. Readhead, W. Xu, and T. J. Pearson

California Institute of Technology, Owens Valley Radio Observatory, 105-24, Pasadena, CA 91125

Received 1994 May 3; accepted 1994 June 28

\begin{abstract}
We present VLBI images of the compact high-luminosity radio galaxy $2352+495$ that show symmetric structure on either side of a prominent central core. This contrasts strongly with the asymmetric nuclear structure exhibited by the great majority of powerful extragalactic sources. The outer structure of $2352+495$ takes the form of two "mini-lobes" containing hot spots; in this respect this compact radio galaxy resembles extended radio galaxies, but its overall size, $\sim 150 \mathrm{pc}$, is $\sim 1000$ times smaller. A reanalysis of existing data on the radio galaxy $0710+439$ shows similar compact structure, and together these VLBI images confirm the existence of a class of two-sided compact symmetric objects (CSOs). We show that, in contrast to nuclear radio sources in other powerful objects, the observed structure of CSOs is not dominated by relativistic beaming effects. It is likely that many objects previously classified as "compact doubles" will prove to be CSOs when mapped with VLBI with high dynamic range.
\end{abstract}

Subject headings: galaxies: active - galaxies: individual $(0710+439,2352+495)$ - galaxies: nuclei radio continuum: galaxies

\section{INTRODUCTION}

Large numbers of radio nuclei have been studied with VLBI over the past 15 years, and it is remarkable how rarely objects with radio emission on both sides of an active core, on scales of 1 to $\sim 100 \mathrm{pc}$, have been found. The first clear example was the radio galaxy $0710+439$ (Readhead, Pearson, \& Unwin 1984; Pearson \& Readhead 1988). Conway et al. (1992) recently confirmed this structure and also drew attention to a similar two-sided radio galaxy, $2352+495$. In this paper we present new VLBI images of $2352+495$ at $\lambda=18 \mathrm{~cm}$ which show that the outer components described by Conway et al. (1992) are in fact hot spots embedded within extended "mini-lobes" straddling the core. We have also reanalyzed previous $\lambda=6 \mathrm{~cm}$ VLBI data on $0710+439$ and found similar extended structure.

The morphology of radio components associated with active galaxies has played a major role in establishing the standard model for these remarkable objects. With this in mind, the discovery of previously elusive symmetric radio structure on the scale of $\sim 100 \mathrm{pc}$ leads us to think that CSOs represent an important type of activity in galactic nuclei and that their study may have far-reaching consequences.

\section{OBSERVATIONS AND DATA REDUCTION}

The observations presented in this Letter are part of our Mark II VLBI study of two flux-density-limited samples of sources selected at $5 \mathrm{GHz}$ : the PR survey of 65 sources with $S_{5 \mathrm{GHz}}>1.3 \mathrm{Jy}$ and $\delta>35^{\circ}$ (Pearson \& Readhead 1988) and the first Caltech-Jodrell Bank or CJ1 survey of 135 sources with $1.3 \mathrm{Jy}>S_{5 \mathrm{GHz}}>0.7 \mathrm{Jy}$ in the same area of sky (Polatidis et al. 1994).

Observations of $2352+495$ at $\lambda=18 \mathrm{~cm}$ were taken with a 12 telescope array in the US ${ }^{1}$ and Europe on 1990 September

\footnotetext{
${ }^{1}$ Including four telescopes of the VLBA. NRAO is operated by Associated Universities, Inc., under cooperative agreement with the National Science Foundation.
}

20; three 30 minute "snapshots" were scheduled. The data were correlated on the 16 station Block2-MkII correlator at Caltech, fringe-fitted in the NRAO AIPS package, and then further analyzed using the Caltech VLBI software package (Pearson 1991). The final maps (Figs. $1 a$ and $1 b$ ) were made with a new "difference mapping" program, DIFMAP (Shepherd, Pearson, \& Taylor 1994). More details of our observations and analysis procedures are given by Polatidis et al. (1994).

The $\lambda=18 \mathrm{~cm}$ map of the galaxy $2352+495$ attracted our attention because the two-sided structure revealed is so different from that of most of the other sources in the survey, the great majority of which are asymmetric core-jets (Wilkinson et al. 1993; Pearson et al. 1994). Recent $\lambda=6 \mathrm{~cm}$ MERLIN observations at a resolution of 50 milliarcsec reveal no further emission around $2352+495$ down to $0.1 \%$ of the peak brightness in the MERLIN map. The overall angular size of $2352+495$ is $\sim 65$ mas, corresponding to $\sim 155 h^{-1}$ pc $\left(H_{0}=\right.$ $\left.100 \mathrm{hm} \mathrm{s}^{-1} \mathrm{Mpc}^{-1} ; q_{0}=0.5\right)$.

Motivated by these new results on $2352+495$, we reanalyzed existing $\lambda=6 \mathrm{~cm}$ VLBI data (epoch 1986.89) on $0710+439$ (Conway et al. 1992) using DIFMAP (see Figs. $2 a$ and $2 b$ ). The resemblance to the images of $2352+495$ is striking, even to the extent of the substructure in the northern component. The southern component has less extended emission but there is low-brightness emission to the east, similar to that in $2352+495$. The angular size of $0710+439$ is $\sim 32$ mas, corresponding to $\sim 110 h^{-1}$ pc. MERLIN observations at $\lambda=6 \mathrm{~cm}$ show that, as with $2352+495$, there is no further emission around $0170+439$ down to $0.1 \%$ of the peak brightness in the MERLIN map.

\section{THE INTEGRATED PROPERTIES OF $0710+439$ AND $2352+495$}

Conway et al. (1992) and Pearson (1993) have drawn attention to the strong similarity between $2352+495$ and $0710+439$ 


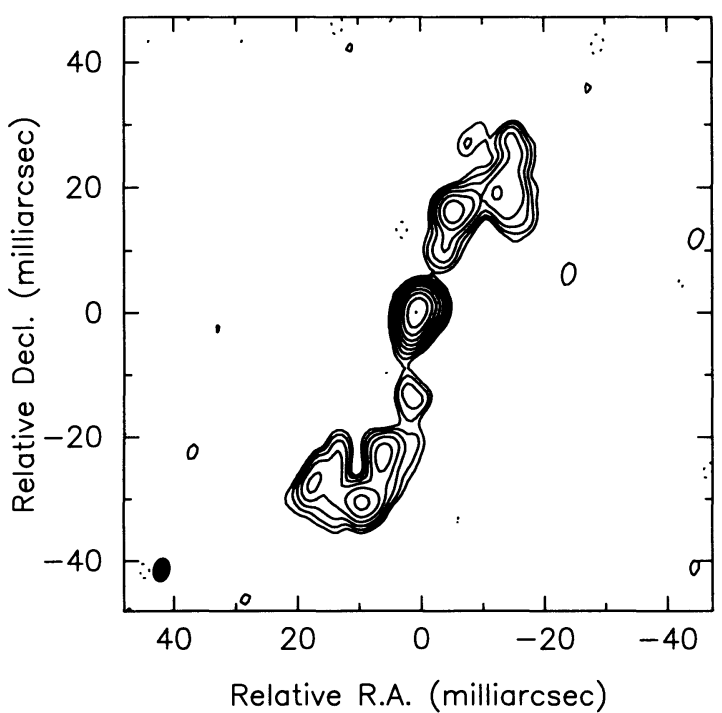

FIG. $1 a$

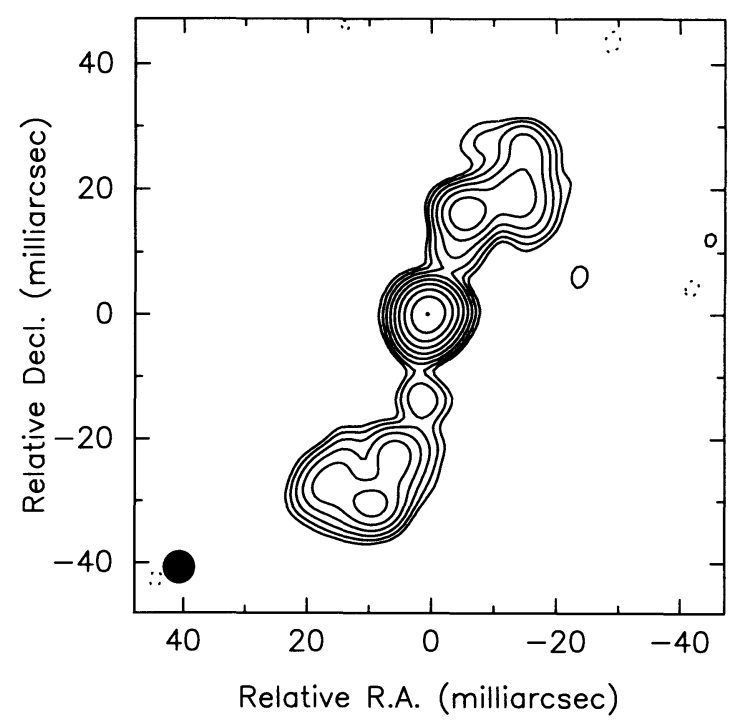

FIG. $1 b$

Fig. 1. (a) VLBI map of $2352+495$ at $\lambda=18 \mathrm{~cm}$ (epoch 1990.72). The data were weighted uniformly, and the restoring beam is $3.64 \times 2.45 \mathrm{mas}$ in p.a. -12.5 . The peak brightness in the map is $664 \mathrm{mJy}$ beam ${ }^{-1}$ and the contours are drawn at $-0.4,0.4,0.8,1.6,3.2,6.4,12.8,25.6$, and $51.2 \%$ of the peak. (b) Map made from the same data, weighted with a Gaussian tapering function $(0.3$ at $30 \mathrm{M} \lambda)$; the restoring beam is 5 mas. The peak brightness in the map is $877 \mathrm{mJy}^{\mathrm{m}}$ beam ${ }^{-1}$ and the contours are drawn at $-0.4,0.4,0.8,1.6,3.2,6.4,12.8,25.6$, and $51.2 \%$ of the peak.

in both optical and radio properties. Both $0710+439$ and $2352+495$ are identified with $\sim 20$ mag galaxies with narrow emission lines and stellar absorption lines but with no broad emission lines or nonthermal continuum. The redshift of $0710+439$ is $z=0.518$ (Lawrence et al. 1986), while that of $2352+495$ is $z=0.237$ (Burbidge \& Crowne 1979). CCD images (O'Dea, Baum, \& Morris 1990; Stanghellini et al. 1993) show that the host galaxies of both radio sources have distorted isophotes and nearby companions. Each appears to be the dominant galaxy in a cluster.

The galaxies $0710+439$ and $2352+495$ have similar integrated radio properties. They have high intrinsic $(\$ 4.2)$ radio luminosities: at an emitted frequency of $5 \mathrm{GHz}$ the monochro-

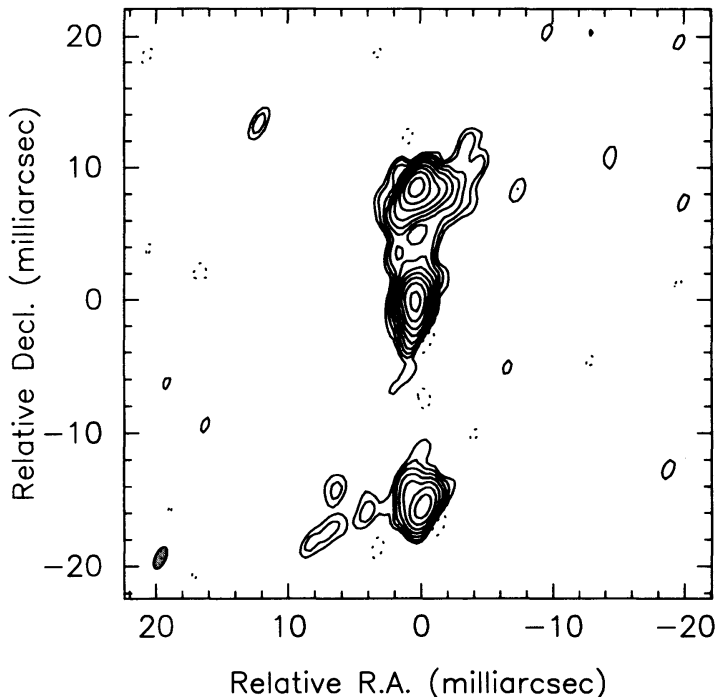

Fig. $2 a$ matic luminosity of $0710+439$ is $5 \times 10^{26} h^{-2} \mathrm{~W} \mathrm{~Hz}^{-1}$, while that of $2352+495$ is $1 \times 10^{26} h^{-2} \mathrm{~W} \mathrm{~Hz}^{-1}$. Note that both objects have monochromatic luminosities much greater than the boundary between F-R I and F-R II radio sources (Fanaroff \& Riley 1974). In the emission rest frame, assuming a spectral index $\alpha=-0.75$, at $5 \mathrm{GHz}$, the F-R I/F-R II boundary occurs at $5 \times 10^{24} h^{-2} \mathrm{~W} \mathrm{~Hz}^{-1}$. Both objects also have low polarization $(\leq 0.2 \%$ at $5 \mathrm{GHz}$; Aller, Aller, \& Hughes 1992) and only low-level flux-density variability (e.g., Seielstad, Pearson, \& Readhead 1983; Aller, Aller, \& Hughes 1992). In each case the integrated spectrum falls off at short wavelengths (Conway et al. 1992), which shows that in these objects there is no major contribution from an active, optically thick core as is

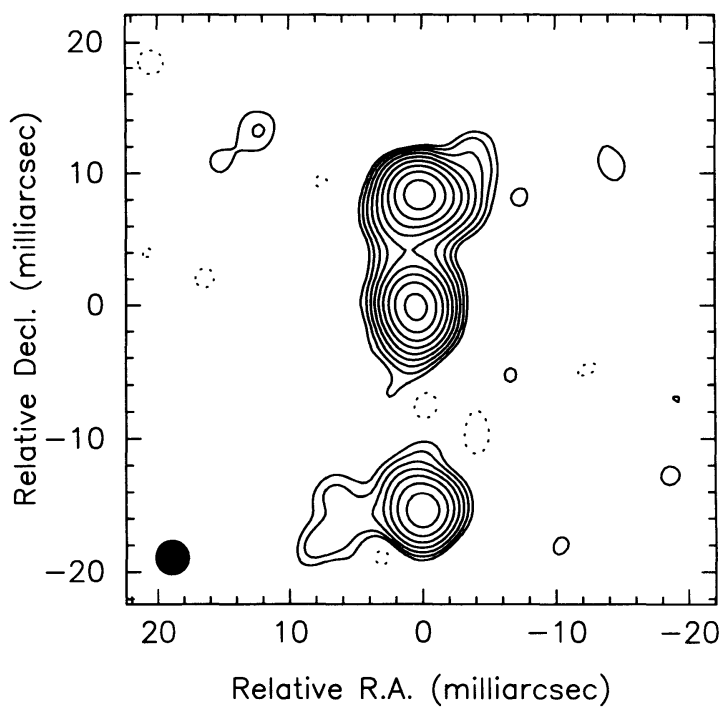

Fig. $2 b$

FIG. 2. (a) VLBI map of $0710+439$ at $\lambda=6 \mathrm{~cm}$ (epoch 1986.89). The data were naturally weighted, and the restoring beam is $1.76 \times 0.81 \mathrm{mas}$ in p.a. $-25^{\circ}$. The peak brightness in the map is $315 \mathrm{mJy}_{\text {beam }}^{-1}$ and the contours are drawn at $-0.25,0.25,0.5,1,2,4,8,16,32$, and $64 \%$ of the peak. (b) Map made from the same data, weighted with a Gaussian tapering function $(0.3$ at $90 \mathrm{M} \lambda)$; the restoring beam is 2.5 mas. The peak brightness in the map is $528 \mathrm{mJy}^{-1}$ beam ${ }^{-1}$ and the contours are drawn at $-0.25,0.25,0.5,1,2,4,8,16,32$, and $64 \%$ of the peak. 
commonly found in radio nuclei studied with VLBI. The spectra also turn down at long wavelengths, showing that the objects do not have significant amounts of optically thin radio emission on scales greater than 0 ". 1 (i.e. a few hundred pc). This has been directly confirmed for $0710+439$ and $2352+495$ (see $\S 2$ ); however, another PR radio galaxy which may belong to the CSO class, $0108+388$ (Conway et al. 1994), does have faint extended structure (Baum et al. 1990).

Overall, many of the integrated properties of $0710+439$ and $2352+495$ are similar to those of the gigahertz-peaked spectrum (GPS) sources (e.g., Gopal Krishna, Patnaik, \& Steppe 1983; O’Dea, Baum, \& Stanghellini 1991).

\section{DISCUSSION}

\subsection{Compact Symmetric Sources}

The structure of $2352+495$ seen in Figure 1 is consistent with the inference of Conway et al. (1992) that the center of activity is located in the middle component. The compact outer components seen by Conway et al. (1992) are now naturally interpreted as hot spots in the outer mini-lobes associated with the current working surfaces of two oppositely directed beams. Readhead et al. (1984) made this same suggestion to account for the structure of $0710+439$, and the case is strengthened by Figure 2. Both outer components of $0710+439$ have extended structure, similar to that seen in our new maps of $2352+495$ but not shown in previous maps.

Few other radio sources have been found with symmetric structure on subkiloparsec scales. Two such sources, in which the core has been unambiguously identified, are in the nuclei of the giant radio galaxies 3C 236 (Barthel et al. 1985; Schilizzi et al. 1988) and 3C 338 (Feretti et al. 1993). However, these twosided nuclear radio sources appear to be fundamentally different from the CSOs. First, their extended structure (on the scale of tens of milliarcseconds, i.e., tens of parsecs) is diffuse and does not show the well-defined lobes and hot spots seen in the CSOs. Second, the luminosity of these nuclear sources is at least two orders of magnitude lower than that of the CSOs. Third, unlike the CSOs, 3C 236 and 3C 338 are dominated by their giant radio lobes rather than their nuclear sources. It seems likely that the axes of 3 C 236 and 3C 338 are very close to the plane of the sky and that the nuclear jets are simply the bases of the two large-scale jets. Both advancing and receding jets could still be visible even if they are relativistic.

Spencer \& Akujor (1992) have discussed other two-sided compact sources; the clearest examples are the quasars $0518+165$ (3C 138), $0538+498$ (3C 147), and $1328+307$ (3C 286). While the luminosities of these sources are similar to those of the CSOs, their structures are different; they do not have the characteristic lobes and hot spots of the CSOs. They are also one to two orders of magnitude larger than the CSOs.

The CSOs are, however, likely to be associated with a subset of the GPS sources, the compact doubles (CDs) (Phillips \& Mutel 1982; Mutel, Hodges, \& Phillips 1985; Hodges \& Mutel 1987) with which they share integrated properties. In their paper reporting the identification of this type of object Phillips \& Mutel (1982) suggested that CDs are symmetric with an unseen central component. However, more recent maps have shown that several apparent CDs are in fact asymmetric corejets $(0153+744$, Hummel et al. 1988; 0711+356 and $2021+614$, Conway et al. 1994), while another is a gravitational lens $(0218+357$; Patnaik et al. 1992). Apparent CDs can also be found by detecting the nucleus and one of the lobes of a
CSO but missing the other lobe; for example, the PR map of $2352+495$ does not include the southern component.

We stress, therefore, that in order to classify an object properly as symmetric on scales of $\sim 100 \mathrm{pc}$, it is essential that the center of activity be identified within the compact radio structure or that other compelling morphological evidence, such as twin jets or outer hot spots, is found. On this basis the early evidence for the two-sided ejection in CDs, based on low dynamic-range VLBI maps, was suggestive but not decisive. Of the 14 objects classified as CDs by O'Dea et al. (1991) and with maps available in the literature, only $1607+628$ shows clear evidence of outer edge-brightening in both components (Mutel et al. 1985).

The VLBI results on $0710+439$ and $2352+495$ reported by Conway et al. (1992) and in this paper do provide decisive evidence for two-sided ejection. The structures of these two objects have now been determined over a sufficiently wide range of frequencies and resolutions to remove any doubt regarding their symmetric nature. We can therefore confirm that a class of powerful compact symmetric radio sources does exist. It is likely that many CDs will be found to be CSOs when mapped with higher dynamic range. Recent VLBI maps suggest that at least three CDs in the O'Dea et al. (1991) compilation $(0026+346$, Taylor et al. $1994 ; 0108+388$, Conway et al. 1994;0404 + 768, Dallacasa et al. 1994, Polatidis et al. 1994, $\mathrm{Xu}$ et al. 1994) contain central cores.

As yet the upper limit in size to CSOs is unclear, largely because the observational study of the flux-limited PR and CJ1 samples is incomplete in the intermediate size range $\sim 0$ ". 1 to $\sim 1^{\prime \prime}$ (i.e., $\sim 300 h$ pc to $\sim 3 h \mathrm{kpc}$ ). In their studies of compact steep spectrum (CSS) sources, Fanti et al. (1990 and references therein) and Dallacasa et al. (1994) have shown that there are some powerful double radio galaxies of intermediate size. These appear to be CSOs significantly larger than those reported here (Fanti et al. 1994).

\subsection{Relativistic Beaming}

Our new results show that relativistic beaming plays at most a minor role in CSOs. First, consider the outer components in $2352+495$ and $0710+439$ : as in large-scale F-R II double sources, their similar brightness and shapes are prima facie evidence against relativistic effects being important, although we cannot rule out beaming by factors of a few. In addition, Conway et al. (1992) failed to detect motion in either $2352+495$ or $0710+439$ with $3 \sigma$ upper limits on the advance speed of the hot spots of $\sim 0.4 h^{-1} c$.

Now consider the compact central components. If, like the majority of powerful sources, they are dominated by jets with large Lorentz factors (i.e., $\gamma \gtrsim 5$ ), the flux density from the central components will be very strongly boosted when the jets are aligned close (i.e., at an angle $\lesssim 1 / \gamma$ ) to the line of sight; this could account for the inclusion of CSOs in flux-limited samples. The population statistics do not, however, support this hypothesis.

If CSOs are preferentially aligned, there must exist an unaligned parent population of objects with weak or undetectable central components; in VLBI surveys these objects would be classed as CDs. If $\gamma>5$, then there must be more than 50 CDs for each CSO observed. In the combined PR and CJ1 samples (a total of 200 objects, of which 127 have been mapped with Mk2 VLBI during the course of our surveys) we have identified nine CSOs (including $0710+439$ and $2352+495$ ) or CSO candidates. The contribution of the 
putative cores ranges from a few percent of the total flux density to $\sim 30 \%$ in the case of $0710+439$ and $\sim 50 \%$ of the total flux density in $2352+495$. It is important to note that all these sources (with $2352+495$ being a marginal case) would be included in the CJ1 survey even if the contribution of the core is neglected. We have found only three candidate compact double sources out of the 127 sources mapped. While objects of intermediate size $\left(\sim 00^{\prime \prime} 1\right.$ to $\sim 1^{\prime \prime}$ arcsec) may have been excluded from our VLBI surveys, we can be certain that there is not a large parent population of CDs. This precludes the possibility of strong relativistic boosting of the central components of CSOs.

This statistical argument against strong boosting is consistent with the detection (Conway et al. 1992) of only subluminal motions within the cores of $0710+439$ and $2352+495$ and with the integrated properties of the two objects. Low polarization, low continuum variability, and lack of components which are optically thick at short wavelengths contrast with the properties of sources which are dominated by relativistically beamed emission. Since relativistic beaming is not a major determining factor, the radio luminosities of CSOs are intrinsically very high. They generate a comparable amount of power to extended F-R II sources but on scales $\sim 1000$ times smaller.

\section{SUMMARY}

The powerful radio galaxy $2352+495$ has a nuclear radio structure comprising two outer "mini-lobes" containing hot spots straddling a central compact core. It appears that the mini-lobes are powered by two oppositely pointing jets emanating from the core. The structure of $2352+495$ is, therefore, similar to that of extended radio galaxies on scales $\sim 1000$ times larger. The nuclear radio structure of the galaxy $0710+439$ strongly resembles that in $2352+495$, and together these results confirm the hypothesis that two-sided ejection is taking place in these objects. While this has long been suspected in the "compact doubles" (e.g., Phillips \& Mutel 1982), the evidence for symmetric ejection on scales of $\sim 100 \mathrm{pc}$ is now unequivocal. We call the class of objects typified by $2352+495$ and $0710+439$ compact symmetric objects (CSOs).

Although our systematic morphological study of CSOs is still in its early stages, we can identify broad defining characteristics, namely, (1) two or more components, separated by $10-1000 \mathrm{pc}$, either straddling a central core or with other compelling morphological evidence for symmetry such as outer hot spots, and (2) no emission on scales greater than $1000 \mathrm{pc}$ or only very faint emission. The morphologies of $0710+439$ and $2352+495$, the population statistics of CSOs and CDs in the PR and CJ1 surveys, and the component proper-motion limits for the core and hot spots derived by Conway et al. (1992) confirm that relativistic beaming does not play a major role in determining the radio properties of CSOs. It is likely that many compact double sources will prove to be CSOs when higher dynamic range VLBI maps are made.

The contrast between the properties of the CSOs and those of the asymmetric core-jet sources seen in most VLBI maps suggests that there are significant physical differences between the jets in CSOs and those in the majority of radio sources. A detailed discussion of the ages and energetics of CSOs and their place in the radio source population is given by Readhead et al. (1994).

We are grateful to the staff at the many observatories who contributed to the success of the first Caltech-Jodrell Bank VLBI survey. This work was supported in part by grants from the National Science Foundation (AST 88-14554 and AST 91-17100).

\section{REFERENCES}

Aller, M. F., Aller, H. D., \& Hughes, P. A. 1992, ApJ, 399, 16

Barthel, P. D., Schilizzi, R. T., Miley, G. K., Jägers, W. J., \& Strom, R. G. 1985, A\&A, 148, 243

Baum, S. A., O’Dea, C. P., Murphy, D. W., \& de Bruyn, A. G. 1990, A\&A, 232, 19

Burbidge, G. H., \& Crowne, A. H. 1979, ApJS, 40, 583

Conway, J. E., Myers, S. T., Pearson, T. J., Readhead, A. C. S., Unwin, S. C., \& $\mathrm{Xu}, \mathrm{W} .1994$, ApJ, 425, 568

Conway, J. E., Pearson, T. J., Readhead, A. C. S., Unwin, S. C., Xu, W., \& Mutel, R. L. 1992, ApJ, 396, 62

Dallacasa, D., Fanti, C., Fanti, R., Schilizzi, R. T., \& Spencer, R. E. 1994, A\&A, submitted

Fanaroff, B. L., \& Riley, J. M. 1974, MNRAS, 167, 31P

Fanti, R., Fanti, C., Stanghellini, C., Schilizzi, R. T., Nan, R., Parma, P., \& van Breugel, W. J. M. 1990, A\&A, 231, 333

Fanti, R., et al. 1994, in preparation

Feretti, L., Comoretto, G., Giovannini, G., Venturi, T., \& Wehrle, A. E. 1993, ApJ, 408, 446

Gopal Krishna, Patnaik, A. R., \& Steppe, H. 1983, A\&A, 199, 73

Hodges, M. W., \& Mutel, R. L. 1987, in Superluminal Radio Sources, ed. J. A. Zenus \& T. J. Pearson (Cambridge: Cambridge Univ. Press), 168

Hummel, C. A., Schalinski, C. J., Krichbaum, T. P., Witzel, A., \& Johnston, K. J. 1988, A\&A, 204, 68

Lawrence, C. R., Pearson, T. J., Readhead, A. C. S., \& Unwin, S. C. 1986, AJ, 91, 494

Mutel, R. L., Hodges, M. W., \& Phillips, R. B. 1985, ApJ, 209, 86

O'Dea, C. P., Baum, S. A., \& Morris, G. B. 1990, A\&AS, 82, 261

O'Dea, C. P., Baum, S. A., \& Stanghellini, C. 1991, ApJ, 380,66

Patnaik, A. R., Browne, I. W. A., King, L. J., Muxlow, T. W. B., Walsh, D., \& Wilkinson, P. N. 1992, MNRAS, 261, 435

Pearson, T. J. 1991, BAAS, 23, 991

. 1993, in Jets in Extragalactic Radio Sources, ed. H.-J. Röser \& K

Meisenheimer (Berlin: Springer-Veriag), 79
Pearson, T. J., \& Readhead, A. C. S. 1988, ApJ, 328, 114 (PR)

Pearson, T. J., Xu, W., Thakkar, D. D., Readhead, A. C. S., Polatidis, A. G., \& Wilkinson, P. N. 1994, in Proc. NRAO Workshop 23, Compact Extragalactic Radio Sources, ed. J. A. Zensus \& K. I. Kellermann (Socorro: NRAO), 1

Phillips, R. B., \& Mutel, R. L. 1982, A\&A, 106, 21

Polatidis, A. G., Wilkinson, P. N., Xu, W., Readhead, A. C. S., \& Pearson, T. J. 1994, ApJS, submitted

Readhead, A. C. S., Pearson, T. J., \& Unwin, S. G. 1984, in IAU Symp. 110, VLBI and Compact Radio Sources, ed. R. Fanti, K. I. Kellermann, \& G. Setti (Dordrecht: Reidel), 131

Readhead, A. C. S., Xu, W., Pearson, T. J., Wilkinson, P. N., \& Polatidis, A. G. 1994 , in preparation

Schilizzi, R. T., Skillman, E. D., Barthel, P. D., Miley, G. K., Benson, J. M., \& Muxlow, T. W. B. 1988, in IAU Symp. 129, The Impact of VLBI on Astrophysics and Geophysics, ed. M. J. Reid \& J. M. Moran (Dordrecht: Kluwer), 127

Seielstad, G. A., Pearson, T. J., \& Readhead, A. C. S. 1983, PASP, 95, 42

Shepherd, M. C., Pearson, T. J., \& Taylor, G. B. 1994, BAAS, 26, 987

Spencer, R. E., \& Akujor, C. E. 1992, in Extragalactic Radio Sources from Beams to Jets, ed. J. Roland, H. Sol, \& G. Pelletier (Cambridge: Cambridge Univ. Press), 119

Stanghellini, C., O’Dea, C. P., Baum, S. A., \& Laurikainen, E. 1993, ApJS, 88, 1

Taylor, G. B., Vermeulen, R. C., Pearson, T. J., Readhead, A. C. S., Henstock, D. R., Browne, I. W. A., \& Wilkinson, P. N. 1994, ApJS, in press

Wilkinson, P. N., Polatidis, A. G., Readhead, A. C. S., Xu, W., \& Pearson, T. J. 1993, in Sub-arcsecond Radio Astronomy (Cambridge: Cambridge Univ. Press), 213

Xu, W., Readhead, A. C. S., Pearson, T. J., Polatidis, A. G., \& Wilkinson, P. N. 1994 , in preparation 